\title{
Desarrollo y elaboración de un snack extruido a partir de quinua (Chenopodium quinoa Willd.) y maíz (Zea mays L.)
}

\author{
Ritva Repo-Carrasco*, Juan Julio Pilco y Christian Rene Encina Zelada \\ Universidad Nacional Agraria La Molina
}

Ingeniería Industrial n²9, 2011, ISSN 1025-9929, pp. 207-224

Recibido: 1 de junio del 2011 / Aprobado: 10 de junio del 2011

\begin{abstract}
RESUMEN: El principal objetivo del presente trabajo de investigación fue desarrollar un producto de snack nutritivo utilizando la quinua y el maíz. Las tres variedades de quinua utilizadas presentaron un alto contenido de proteínas y fibra dietaria, así como compuestos fenólicos y capacidad antioxidante. La mejor variedad para el proceso de extrusión fue la chullpi y la mezcla óptima fue $70 \%$ de quinua y $30 \%$ de maíz. Este producto ofrece una alternativa nutritiva a los snacks tradicionales.
\end{abstract}

Palabras claves: extrusión / quinua / snacks / cubierta dulce / maíz / valor nutricional / Perú

\section{Development of an extruded snack using quinoa (chenopodium quinoa willd) and corn (zea mayz l)}

ABstRACT: The objective of this study was to develop a nutritious snack using a mixture of quinoa and corn. All the three varieties had high protein and dietary fiber content and were rich in polyphenolic compounds and antioxidants. The best variety for extrusion was Chullpi and the most suitable mixture was 70\% quinoa, Chullpi variety, and 30\% corn. The common snacks preferred by children and teenagers are rich in calories and have few nutrients, thus this product offers an excellent nutritive alternative to traditional snacks.

Keywords: extrusion / quinoa / snacks / sweet coverage / corn / nutritional value / Perú

* Autor responsable. 


\section{INTRODUCCIÓN}

Como tecnología de punta revolucionaria en la industria alimentaria, la extrusión ofrece ventajas económicas, nutricionales y productivas muy tentadoras cuando se requiere brindar alimentos nutritivos, de conveniencia y a su vez dar rentabilidad y ganancias sustanciales (Ramos 2002). La cocción-extrusión con equipos de bajo costo es un tipo de procesamiento de operación que sumado a su mínima complejidad resulta viable para ser transferido a zonas altoandinas para su aplicación en la preparación de mezclas alimenticias. La extrusión no es un proceso sencillo.

La elaboración de productos expandidos mediante el empleo de extrusores presenta grandes ventajas, puesto que no utiliza grasa durante la cocción, el producto snack mantiene las propiedades químicas de la materia prima, emplea menor cantidad de materia prima que otros procesos alternativos y sobre todo es un método rápido. Sin embargo, el proceso de extrusión eleva la temperatura, por lo cual puede degradar los componentes. Por otro lado, el producto final es de buena calidad y de bajo costo (Sandoval 1993).

Esta alternativa tecnológica nos ofrece la oportunidad de aprovechar de la mejor manera la producción de cereales en las zonas altoandinas de nuestro país; de esta forma se podrá proporcionar un producto con alto valor nutritivo que contribuya a resolver el problema de desnutrición en nuestro país, además de brindar una alternativa comercial para el mercado de golosinas.

La quinua (Chenopodium quinoa Willd) es un grano alimenticio que se cultiva ampliamente en la región andina, desde Colombia hasta el norte de la Argentina, por las condiciones que ofrecen las montañas de altura, pero también existe un ecotipo que se produce en Chile a nivel del mar. En la actualidad en el Perú es donde más se cultiva la quinua y donde se ha seleccionado una serie de variedades (Tapia 1997).

El grano de quinua posee cualidades nutritivas bastante destacables, que radican principalmente en la calidad de su proteína, tanto en el cómputo aminoacídico como en el valor biológico que esta presenta. Su fracción lipídica es también importante, debido a la presencia de varios ácidos grasos esenciales (Repo-Carrasco 1998).

La presente investigación tuvo los siguientes objetivos: determinar la composición química proximal de tres variedades de quinua cruda, 
determinar la proporción quinua/maíz que presente el índice más alto de expansión y el mayor grado de gelatinización en el proceso de cocción-extrusión, y obtener un producto tipo snack con cobertura dulce, a partir de la quinua (Chenopodiun quinoa Willd) y el maíz (Zea mays L.) mediante el proceso de cocción-extrusión y la caracterización físicoquímica del producto terminado.

\section{MATERIALES Y MÉTODOS}

\subsection{Materiales}

Los materiales empleados fueron: tres variedades de quinua (Chenopodiun quinoa Willd), procedentes del departamento de Puno; maíz amiláceo, proveniente del Cusco; azúcar blanca refinada, azúcar impalpable y limón sutil.

\subsection{Equipo}

El equipo estuvo compuesto por los siguientes implementos: molino de martillo Vulcano, tamiz vibratorio RO-TAP JEL, tamices $\mathrm{N}^{\circ} 10,16$, 18 y 20 de la US Standar ASTM, W.S. Tyler, extrusor de bajo costo, grageadora, estufas Lämpökaappi 10 serie 30051 y Jermaks serie T8 4115 .

\subsection{Metodología experimental}

En lo que se refiere a la metodología experimental, se siguieron los pasos que a continuación ennumeramos:

- Obtención de granos de quinua desaponificados.- Se realizó una limpieza manual con el fin de eliminar piedrecillas, paja y granos deteriorados. Se efectuaron lavados consecutivos con agua a $50^{\circ} \mathrm{C}$, con agitación constante hasta que dejó de formar espuma. Se secó en una estufa a $45^{\circ} \mathrm{C}$ por 12 horas y se acondicionó hasta alcanzar el $11 \%$ de humedad.

- Obtención de gritz de maíz.- Se hizo una limpieza manual con la finalidad de eliminar piedrecillas, pajas y granos dañados o picados. La molienda se realizó en un molino de martillos; el producto 
obtenido fue tamizado con malla $\mathrm{N}^{\circ} 16$ de la U.S. Standar ASTM y acondicionado hasta alcanzar el 11\% de humedad.

- Proceso de extrusión.- Se realizó con el fin de cocer, formar, texturizar y deshidratar las diferentes formulaciones. Se utilizó un extrusor de bajo costo, todas las formulaciones fueron extruidas a $160^{\circ} \mathrm{C}$, velocidad de tornillo igual a $254.5 \mathrm{rpm}$ y dos orificios de salida de $3 / 16$ pulgadas.

- Determinación de la mezcla óptima.- Se hicieron cinco mezclas entre los granos de quinua de la variedad choclito de Ayaviri y los gritz de maíz (proporciones de 1:1 hasta 9:1, respectivamente), cada una de ellas fue humectada a tres niveles de humedad (12, 14 y 16\%). Los productos extruidos obtenidos de cada tratamiento fueron evaluados en sus características funcionales, siendo el índice de expansión y el grado de gelatinización los que determinaron la mejor mezcla. Posteriormente, se realizaron mezclas con cada una de las variedades de quinua para evaluar la influencia de la variedad sobre el producto extruido, tomando en cuenta la formulación determinada anteriormente. Los productos obtenidos fueron evaluados en sus características funcionales, siendo el índice de expansión y el grado de gelatinización las que determinaron la mejor variedad.

- Elaboración de la cobertura dulce.- Se elaboró un almíbar de $70^{\circ}$ Brix, mezclando agua de mesa y azúcar blanca refinada hasta alcanzar los $100^{\circ} \mathrm{C}$, luego se agregó zumo de limón $(5 \mathrm{ml} / 100 \mathrm{ml}$ de almíbar).

- Obtención del snack con cobertura dulce.- Una vez determinada la mezcla óptima se procedió a elaborar los snacks recubriendo el producto con una solución azucarada de $70^{\circ}$ Brix, saborizada con zumo de limón $\left(5 \mathrm{ml} / 100 \mathrm{ml}\right.$ de almíbar) a $100^{\circ} \mathrm{C}$ y posteriormente se dejó secar a $60^{\circ} \mathrm{C}$ por 24 horas.

\subsection{Métodos de análisis}

A continuación se describen los métodos de análisis empleados:

- Análisis de la composición próxima.- Agua, proteína ( $\mathrm{N}$ x 6.25), grasa, fibra cruda y cenizas fueron determinadas de acuerdo con la AOAC (1995). El contenido de carbohidratos (CHO) fue calculado por diferencia mediante la siguiente relación: $\mathrm{CHO}=100$ - (agua + proteína + grasa + fibra cruda + ceniza). 
- Determinación de la capacidad antioxidante y compuestos fenólicos.Capacidad antioxidante por el método ABTS (Arnao 2001: 419-421); polifenoles totales (Swain y Hillis 1959: 63-68).

- Características funcionales del extruido.- Índice de solubilidad en agua (ISA), índice de absorción de agua (IAA) y grado de gelatinización (Casas 1996); densidad (Muller 1978) e índice de expansión (Casas 1996).

- Evaluación sensoria.- Prueba de nivel de agrado de nueve puntos (Hedonic Test) (Pedrero y Marie 1996); prueba de aceptación (Pedrero y Marie 1996).

- Análisis estadístico.- Los análisis se trabajaron por triplicado, indicando el promedio \pm desviación estándar. Para la determinación del efecto de la variedad de quinua sobre las determinaciones, se hizo un arreglo factorial evaluado con un diseño completamente al azar (DCA) para los diferentes análisis realizados, aplicando un análisis de varianza (Anova), y cuando hubo diferencias significativas (con un nivel de significancia del 95\%), se procedió a efectuar un test de comparación múltiple aplicando la prueba de Tukey $(p<0,05)$. El análisis estadístico se realizará con el paquete Statgraphics Centurion.

\section{RESULTADOS Y DISCUSIÓN}

\subsection{Composición proximal de las materias primas}

Al comparar los resultados del análisis proximal de las tres variedades de quinua, presentados en el cuadro 1, se pueden observar diferencias importantes entre los contenidos de algunos componentes, como el contenido proteico, la grasa y la fibra cruda. Al respecto, Mujica et al. (2001), en un trabajo para la FAO, reportaron valores de composición proximal para la quinua: $10,05 \%, 13,7 \%, 5,1 \%, 2,7 \%, 4,45 \%$ y $69,7 \%$, para humedad, proteína, grasa, ceniza, fibra y carbohidratos, respectivamente. Aguirre (2003) reporta datos promedio de cinco autores para humedad, proteína, grasa, ceniza, fibra y carbohidratos, siendo la composición proximal de la quinua: $11,6 \%, 15,1 \%, 6,2 \%, 3,2 \%, 3,9 \%$ y $62,4 \%$. 


\section{Cuadro 1}

Composición proximal de tres variedades de quinua cruda y del maíz amiláceo (b.s.)

\begin{tabular}{lcccc}
\hline \multirow{2}{*}{ Componente } & \multicolumn{3}{c}{ Variedad } \\
\cline { 2 - 4 } & Choclito de Ayaviri & Chullpi & Pasankalla & Maíz \\
\hline Humedad $^{*}$ & $8,93 \pm 0,08$ & $9,99 \pm 0,11$ & $9,74 \pm 0,07$ & $10,84 \pm 0,08$ \\
Proteínas $^{\text {a }}$ & $12,88 \pm 0,19$ & $13,29 \pm 0,11$ & $14,09 \pm 0,11$ & $5,68 \pm 0,01$ \\
Grasa & $6,78 \pm 0,15$ & $7,52 \pm 0,76$ & $7,05 \pm 0,16$ & $2,21 \pm 0,00$ \\
Cenizas & $3,19 \pm 0,11$ & $2,65 \pm 0,10$ & $3,25 \pm 0,20$ & $1,93 \pm 0,01$ \\
Fibra cruda $_{\text {Carbohidratos }^{b}}$ & $2,45 \pm 0,28$ & $2,32 \pm 0,06$ & $2,86 \pm 0,05$ & $0,039 \pm 0,00$ \\
& 74,70 & 74,22 & 72,75 & 90,14 \\
\hline
\end{tabular}

* b.h.;

a $\% \mathrm{~N}$ x 6.25;

b por diferencia.

Elaboración propia.

\section{Figura 1}

Variedades de quinua empleadas en la investigación

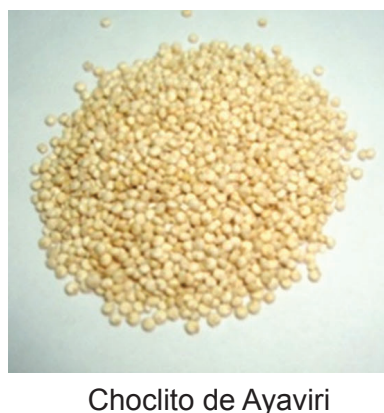

Choclito de Ayaviri

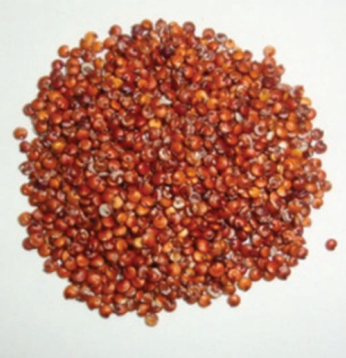

Pasankalla

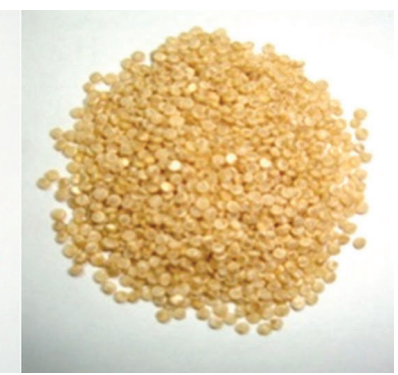

Chullpi

Elaboración propia.

La variación de los componentes de la quinua puede deberse a varios factores, como variedad, clima, suelo, etcétera; por ello, la composición proximal de cada variedad difiere una de otra.

Por otro lado, el maíz presenta un elevado contenido de carbohidratos. Collazos et al. (1996) encontraron un contenido de 74,30\% para la variedad amilácea (INIA 607 - Checche andenes), mientras que en la investigación se determinó un contenido de $80,38 \%$ b.h. y $90,14 \%$ b.s., superior al que aparece en la bibliografía citada. 


\subsection{Determinación de la capacidad antioxidante y compuestos fenólicos de las materias primas}

Se determinó el contenido de compuestos fenólicos o polifenoles totales en las tres variedades de quinua usadas en la investigación (cuadro 2). La variedad Pasankalla presentó el mayor contenido de compuestos fenólicos totales. De acuerdo con la prueba de comparación de Tukey $(p<0,05)$, las tres variedades muestran diferencias estadísticamente significativas entre sí.

Bressani (1993: 123-143) menciona que el contenido de compuestos fenólicos varía de acuerdo con la coloración de la cáscara; encontrando en sus análisis frijoles marrones, negros, rojos y blancos, valores de 7,$8 ; 6,6 ; 12,6$ y $2,3 \mathrm{mg} / \mathrm{g}$ de equivalentes de catequina, respectivamente; por lo que las diferencias del contenido de polifenoles totales en cada variedad de quinua analizada también están relacionadas con su color. Esto se ve reflejado en la figura 1, donde la variedad Chullpi presenta el menor contenido de polifenoles totales $(69,71 \mathrm{mg}$ ácido gálico/100 g b.h.), considerando que esta variedad posee granos de color amarillento translúcido, mientras que la variedad Choclito de Ayaviri presentó un contenido superior (75,67 mg ácido gálico/100 g b.h.) siendo una variedad con granos de color blanco, el mayor valor obtenido fue por la variedad Pasankalla (76,79 mg ácido gálico/100 g b.h.), que presenta granos de color rojizo, más oscuro que las anteriores.

\section{Cuadro 2}

Contenido de polifenoles totales y capacidad antioxidante de tres variedades de quinua cruda

\begin{tabular}{lccc}
\hline \multicolumn{1}{c}{ Variedad } & $\begin{array}{c}\text { CPT } \\
\text { (mg ácido clorogénico } \\
\text { /100 g b.h.) }\end{array}$ & $\begin{array}{c}\text { CPT } \\
\text { (mg ácido gálico } \\
\text { /100 g b.h.) }\end{array}$ & $\begin{array}{c}\text { CA } \\
\text { (ug Trolox/g) }\end{array}$ \\
\hline Choclito de Ayaviri & $187,86 \pm 0,88$ & $75,67 \pm 0,30$ & $2133,38 \pm 7,43$ \\
Chullpi & $170,60 \pm 0,67$ & $69,71 \pm 0,23$ & $1835,11 \pm 9,73$ \\
Pasankalla & $191,08 \pm 0,25$ & $76,79 \pm 0,09$ & $2624,54 \pm 10,12$ \\
\hline
\end{tabular}

CPT: Contenido de polifenoles totales

CA: Capacidad antioxidante

Elaboración propia 
Awika et al. (2003: 6657-6662) mencionan que el ABTS constituye una mejor opción frente al DPPH cuando se trata de analizar la capacidad antioxidante, puesto que es un método de alta flexibilidad y puede ser usado a diferentes niveles de $\mathrm{pH}$, en contraposición al DPPH, que es sensible a $\mathrm{pH}$ ácidos, siendo poco útil su aplicación cuando se desea evaluar el efecto del $\mathrm{pH}$ en la actividad antioxidante de varios compuestos.

De acuerdo con la prueba de comparación de Tukey $(\mathrm{p}<0,05)$ las tres variedades muestran diferencias estadísticamente significativas entre sí, siendo la variedad Pasankalla la que presentó la mayor capacidad antioxidante (2624,54 $\mu \mathrm{g}$ Trolox/g), seguido de la variedad Choclito de Ayaviri (2133,38 $\mu \mathrm{g}$ Trolox/g) y finalmente la variedad Chullpi (1835,11 $\mu \mathrm{g}$ Trolox/g).

Los antioxidantes son compuestos que inhiben o retrasan la oxidación de otras moléculas mediante la inhibición de la propagación de la reacción de oxidación. Los compuestos fenólicos son antioxidantes naturales, por lo que la capacidad antioxidante está directamente relacionada con el contenido de polifenoles totales. En este estudio se encontró una correlación positiva entre polifenoles totales y capacidad antioxidante en las tres variedades de quinua estudiadas.

\subsection{Determinación de la mezcla óptima y del producto final}

Los resultados de la evaluación de los productos obtenidos al aplicar los distintos tratamientos se presentan en el cuadro 3.

Se eligió como mejor tratamiento el $\mathrm{N}^{\circ} 8$, con un Proporción quinua:maíz de 70:30 y Humedad de alimentación de 14\%. Este tratamiento presentó el mayor ISA $(0,44)$, el segundo mayor IAA $(6,90)$, la menor densidad $(0,07 \mathrm{~g} / \mathrm{ml})$, el mayor índice de expansión $(2,49)$ y el mayor grado de gelatinización $(68,22 \%)$, siendo estas dos últimas variables de respuesta las que determinaron la elección debido a que el índice de expansión controla la relación existente entre la densidad global y la textura del producto extruido (Guy 2002), características muy importantes desde el punto de vista comercial y sensorial, respectivamente. 


\section{Cuadro 3}

Resultados del análisis de los extruidos de cada uno de los 15 tratamientos

\begin{tabular}{|c|c|c|c|c|c|c|c|c|c|}
\hline $\mathbf{N}^{\circ}$ Trat. & $\begin{array}{c}\text { Quinua } \\
\text { (\%) }\end{array}$ & $\begin{array}{c}\text { Maíz } \\
(\%)\end{array}$ & $\begin{array}{c}H \\
(\%)\end{array}$ & Maquinabilidad & ISA & IAA & $\begin{array}{c}\text { Densidad } \\
(\mathrm{g} / \mathrm{ml})\end{array}$ & $\begin{array}{l}\text { Índice de } \\
\text { expansión }\end{array}$ & $\begin{array}{c}\text { Grado de } \\
\text { gelatinización } \\
(\%)\end{array}$ \\
\hline 1 & 50 & 50 & 12 & $\checkmark$ & 0,27 & 6,83 & 0,10 & 1,33 & 58,36 \\
\hline 2 & 50 & 50 & 14 & $\checkmark$ & 0,16 & 7,84 & 0,15 & 1,72 & 53,02 \\
\hline 3 & 50 & 50 & 16 & $x$ & - & - & - & - & - \\
\hline 4 & 60 & 40 & 12 & $x$ & - & - & - & - & - \\
\hline 5 & 60 & 40 & 14 & $\checkmark$ & 0,26 & 6,86 & 0,09 & 1,92 & 57,68 \\
\hline 6 & 60 & 40 & 16 & $\checkmark$ & 0,22 & 6,78 & 0,14 & 1,97 & 54,76 \\
\hline 7 & 70 & 30 & 12 & $x$ & - & - & - & - & - \\
\hline $8^{*}$ & 70 & 30 & 14 & $\checkmark$ & 0,44 & 6,90 & 0,07 & 2,49 & 68,22 \\
\hline 9 & 70 & 30 & 16 & $\checkmark$ & 0,34 & 6,35 & 0,11 & 2,16 & 59,04 \\
\hline 10 & 80 & 20 & 12 & $x$ & - & - & - & - & - \\
\hline 11 & 80 & 20 & 14 & $\checkmark$ & 0,41 & 6,65 & 0,09 & 2,22 & 64,50 \\
\hline 12 & 80 & 20 & 16 & $\checkmark$ & 0,25 & 6,17 & 0,21 & 1,86 & 58,65 \\
\hline 13 & 90 & 10 & 12 & $x$ & - & - & - & - & - \\
\hline 14 & 90 & 10 & 14 & $\checkmark$ & 0,36 & 6,88 & 0,15 & 1,78 & 59,98 \\
\hline 15 & 90 & 10 & 16 & $x$ & - & - & - & - & - \\
\hline
\end{tabular}

$\% \mathrm{H}:$ Porcentaje de humedad

$\checkmark$ Arrastre de materia prima adecuada

$x$ Arrastre de materia prima inadecuada
ISA: Índice de solubilidad en agua IAA: Índice de absorción de agua

* Mejor tratamiento

Elaboración propia.

Aguirre (2003) usó el índice de expansión como un requisito para determinar los parámetros de extrusión óptimos para la quinua, siendo considerados los valores mayores a 2,40 como aceptables; en la presente investigación se obtuvo un índice de expansión máximo de 2,49. Asimismo, el grado de gelatinización del almidón es muy importante, ya que realza la apariencia, el sabor, la textura y la digestibilidad de los alimentos (Manrique et al. 2007: 627-635). 
Además, al maximizar estadísticamente los valores de estas dos variables se determinó que los valores máximos se obtenían, en promedio, con $71,98 \%$ de quinua en la mezcla y $13,43 \%$ de humedad de alimentación, por lo que el tratamiento que presentaba las variables operativas más cercanas y las variables de respuesta más altas (figuras 2 y 3) fue el elegido.

Figura 2

Gráfica de efectos principales para el Índice de expansión

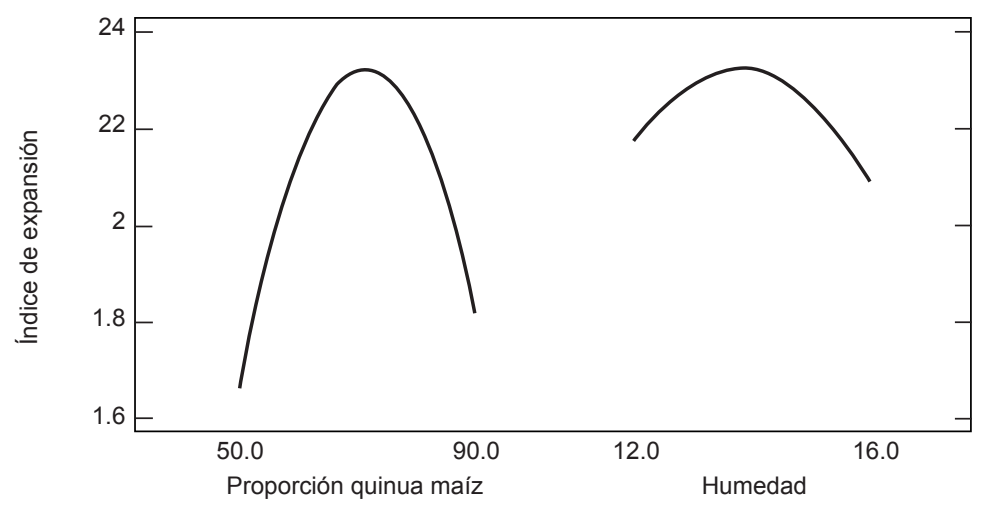

Elaboración propia.

Figura 3

Gráfica de efectos principales para el grado de gelatinización

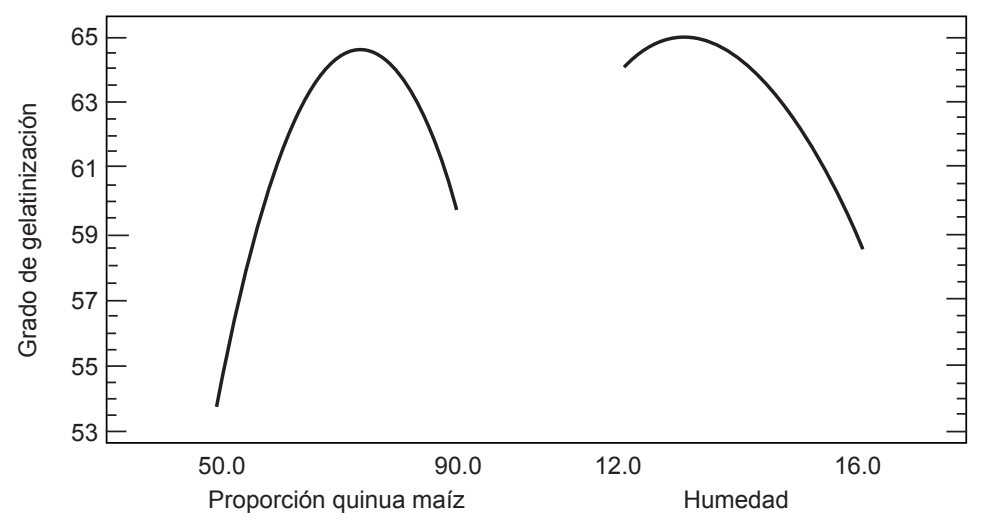

Elaboración propia. 
Una vez determinada la mezcla y el porcentaje de humedad óptimo, se procedió a evaluar la influencia de la variedad sobre el producto. Los resultados obtenidos en la investigación (cuadro 4) y su análisis estadístico indican que la variedad de quinua es un factor que tiene un efecto estadísticamente significativo en las características del producto extruido, lo cual se refleja en las diferencias significativas que presentaron las variables de respuesta estudiadas.

La variedad que brindó mejores resultados fue la variedad Chullpi. El producto obtenido a partir de esta variedad posee el mayor ISA entre las tres variedades $(0,28)$, el menor IAA $(6,34)$, la menor densidad $(0,04 \mathrm{~g} / \mathrm{ml})$, el mayor índice de expansión $(2,87)$ y el mayor grado de gelatinización $(81,24 \%)$, siendo estas dos últimas características las que determinaron su elección como la mejor variedad.

\section{Cuadro 4}

Resultados del análisis de los extruidos de las tres variedades de quinua

\begin{tabular}{lcccccc}
\hline Variedad & Maquinabilidad & ISA & IAA & $\begin{array}{c}\text { Densidad } \\
\mathbf{( g / m l )}\end{array}$ & $\begin{array}{c}\text { Índice de } \\
\text { expansión }\end{array}$ & $\begin{array}{c}\text { Grado de } \\
\text { gelatinización } \\
\mathbf{( \% )}\end{array}$ \\
\hline Choclito de Ayaviri & $\checkmark$ & 0,21 & 6,92 & 0,11 & 2,59 & 73,78 \\
Chullpi $^{*}$ & $\checkmark$ & 0,28 & 6,34 & 0,04 & 2,87 & 81,24 \\
Pasankalla & $\checkmark$ & 0,22 & 6,58 & 0,10 & 2,21 & 67,79 \\
\hline
\end{tabular}

\section{* Mejor variedad}

Elaboración propia

Una vez elegida la mezcla óptima se procedió a adicionar la cobertura dulce a base de sacarosa $\left(70^{\circ} \mathrm{Brix}\right)$ haciendo uso de una grageadora, luego el producto obtenido fue secado a $60^{\circ} \mathrm{C}$ por 24 horas para alcanzar una humedad menor a $6 \%$. Adicionalmente, se espolvoreó azúcar impalpable a un grupo de ellos.

\subsection{Evaluación sensorial}

La evaluación de las muestras fue por separado. En la primera prueba, las muestras fueron evaluadas en sus atributos de color, olor, sabor y aceptabilidad general; en esta prueba los panelistas ubicaron cada 
muestra dentro de una escala de nueve puntos, según su nivel de agrado. En la segunda prueba los panelistas tenían que señalar si aceptaban o no el producto; es decir, si lo consumirían si estuviese disponible en el mercado. Los resultados de ambas pruebas se grafican en las figuras 4 y 5 .

\section{Figura 4}

Nivel de agrado promedio de los extruidos con cobertura dulce $\mathbf{n}=\mathbf{8 0}$ panelistas; las letras iguales indican que no hay diferencia significativa

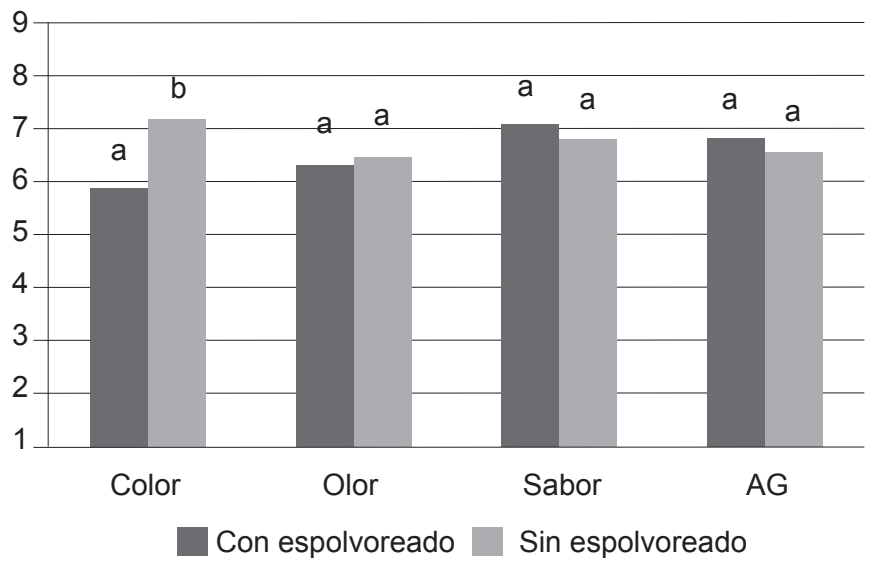

Elaboración propia.

Figura 5

Porcentaje de aceptación del extruido con cobertura dulce y espolvoreada $\mathrm{n}=\mathbf{8 0}$ panelistas $(100 \%)$

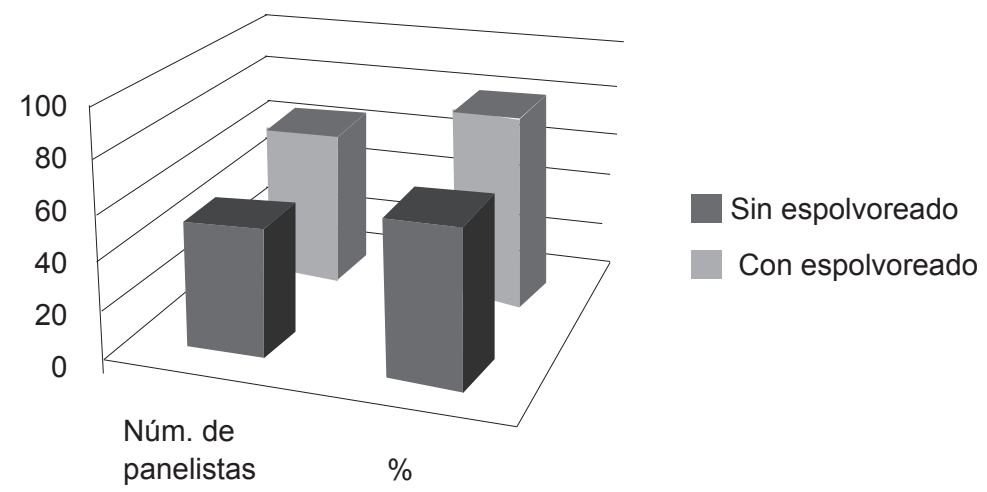

Elaboración propia. 
En la prueba de nivel de agrado, como se observa en la figura 4, no se encontraron diferencias significativas en cuanto al olor, sabor y aceptabilidad general de los productos; sin embargo, sí existe diferencia significativa en cuanto al nivel de agrado del color de los productos, teniendo un nivel de agrado mayor el extruido sin espolvorear, esto debido a que el azúcar impalpable otorga un color blanquecino al producto; en cambio la cobertura dulce sola le otorga al producto un color dorado muy agradable a la vista.

Por otro lado, el producto espolvoreado obtuvo un nivel de agrado ligeramente superior al producto no espolvoreado con respecto al sabor; sin embargo, estadísticamente no existe una diferencia significativa entre ambos productos.

Respecto a la prueba de aceptación, ambos productos tuvieron un porcentaje de aceptación mayor de 50\%; sin embargo, el producto que obtuvo un porcentaje de aceptación mayor fue el espolvoreado, con aceptación por parte de los panelistas (figura 5). Si bien la prueba de nivel de agrado nos permitió identificar que existen diferencias significativas en cuanto al color del producto, la prueba de aceptación nos indica que, aun teniendo un nivel de agrado menor respecto a esta característica, el producto espolvoreado tiene un porcentaje de aceptación significativamente mayor que el producto sin espolvorear.

\section{CONCLUSIONES}

- Las tres variedades de quinua estudiadas presentaron diferencias estadísticamente significativas en su composición de humedad, proteína, ceniza y fibra pero no en su contenido de grasa.

- Las tres variedades de quinua presentan un alto contenido proteico, siendo la variedad Pasankalla la de mayor contenido, con 14,09 \pm $0,19 \%$, seguida de la variedad Chullpi con $13,29 \pm 0,11 \%$, y finalmente la variedad Choclito de Ayaviri, con $12,88 \pm 0,11 \%$ en base seca.

- Las tres variedades de quinua poseen una alta capacidad antioxidante y contenido de polifenoles totales, siendo la más elevada la de la variedad Pasankalla.

- La mezcla a extruir con una "proporción quinua : maíz" de 70:30 y una humedad de alimentación de 14\% presentó el mayor índice de 
expansión $(2,49)$ y el mayor grado de gelatinización $(68,22 \%)$, por lo que fue elegida como la mejor mezcla.

- La mezcla con la variedad Chullpi ofrece mejores características funcionales y principalmente el mayor índice de expansión $(2,87)$ y el mayor grado de gelatinización $(81,24 \%)$ entre las tres variedades de quinua, por lo que fue elegida como la mejor variedad.

- El producto extruido tipo snack, con cobertura dulce y espolvoreada, fue elegido como el mejor, después del análisis sensorial, teniendo como parámetro principal en la determinación la mayor inclusión de quinua y el mayor grado de aceptación.

- Esta investigación demuestra que la quinua es una excelente opción como materia prima para ser usada en productos extruidos elevando su valor nutricional.

\section{BIBLIOGRAFÍA}

Aguirre, J. (2003). "Utilization of the technology of extrusion cooking of low cost in Quinoa processing (Chenopodium quinoa Willd)". Tesis para optar el título de ingeniero de Industrias Alimentarias. Lima: Universidad Nacional Agraria La Molina.

Arnao, M. (2000). "Some methodological problems in the determination of antioxidant activity using cromogen radicals: A practical case". Trends in Food Science and Technology. Vol. 2. Elsevier B. V.

Association of Official Analytical Chemists (AOAC) (1995). Official methods of analysis. 15. ${ }^{a}$ edición. Washington D. C.: Association of Official Analytical Chemists.

Astuhuaman, L. (2007). "Efecto de la cocción-extrusión en la fibra dietaria y en algunas propiedades funcionales de 4 variedades de quinua (Chenopodium quinoa Willd.)". Tesis para optar el título de ingeniero de Industrias Alimentarias. Lima: Universidad Nacional Agraria La Molina.

Awika, J. et al. (1993). "Screening method to measure antioxidant activity of sorghum (Sorghum bicolor) and sorghum products". Journal of Agriculture and Food Chemistry. Vol. 51. Washington D. C.: ACS Publications. 
Bressani, R.; De Martell, E. and Godinez, C. (1993). "Protein quality evaluation of amaranth in adult humans". Plant Foods for $\mathrm{Hu}$ man Nutrition. Vol. 43, núm. 2. Netherlands: Kluwer Academic Publishers.

Casas, J. (1996). "Evaluación de los parámetros de extrusión de una mezcla de harina de habas y maíz usando el método de superficie de respuesta". Tesis para optar el título de ingeniero de Industrias Alimentarias. Lima: Universidad Nacional Agraria La Molina.

Guy, R. (2002). "Extrusion cooking: Technologies and applications". Woodhead Publishing Limited. Washington D. C.: CRC Press.

Manrique, N. et al. (2007). "Caracterización de mezclas de almidones de mango y plátano pregelatinizados mediantes diferentes condiciones de extrusión". Agrociencia. Vol. 41. México.

Mujica, A. et al. (2001). "Quinua (Chenopodium quinoa Willd): Cultivo andino, alimento presente y futuro". <http://www.rlc.fao.org/>. Santiago de Chile: Oficina Regional de la FAO para América Latina y el Caribe. [Consulta: 4 de febrero del 2009.]

Muller, H. (1978). Introducción a la reología de los alimentos. Zaragoza: Acribia.

Pedrero, F. y R. Marie (1996). Evaluación sensorial de los alimentos. Métodos analíticos. México D. F.: Alhambra Mexicana.

Ramos, L. (2002). "Aspectos tecnológicos para la extrusión de cereales andinos". Monografía. Lima: Universidad Nacional Agraria La Molina.

Repo-Carrasco, R. (1998). Introducción a la ciencia y tecnología de cereales y granos andinos. Lima: Universidad Nacional Agraria La Molina.

Sandoval, N. (1993). "Elaboración de un producto tipo snack (bocaditos) a partir de la mezcla de harinas de maíz (Zea mays L.) y pituca (Colocasia esculenta)". Tesis para optar el título de ingeniero de Industrias Alimentarias. Lima: Universidad Nacional Agraria La Molina. 
Swain, T. y W. Hills (1959). "The phenolic constitutes of prunus domestica. I The quantitative analysis of phenolic constituents". Journal of Food Science. Vol. 10. Chicago: Institute of Food Technologists.

Tapia, M. (1997). Cultivos andinos subexplotados y su aporte a la alimentación. Santiago de Chile: Oficina Regional de la FAO para América Latina y el Caribe. 